\title{
One-dimensional isothermal multicomponent diffusion-reaction model and its application to methanol synthesis over commercial Cu-based catalyst
}

\author{
Kun Lei, Hongfang Ma*, Haitao Zhang, Weiyong Ying, Dingye Fang \\ East China University of Science and Technology, Engineering Research Center of Large Scale Reactor Engineering and \\ Technology, Ministry of Education, State Key Laboratory of Chemical Engineering, Po Box 374, NO.130 Meilong Road, \\ Shanghai 200237, China \\ "Corresponding author: e-mail: mark@ecust.edu.cn
}

\begin{abstract}
The present work was a study on global reaction rate of methanol synthesis. We measured experimentally the global reaction rate in the internal recycle gradientless reactor over catalyst SC309. The diffusion-reaction model of methanol synthesis was suggested. For model we chose the hydrogenation of $\mathrm{CO}$ and $\mathrm{CO}_{2}$ as key reaction. $\mathrm{CO}$ and $\mathrm{CO}_{2}$ were key components in our model. The internal diffusion effectiveness factors of $\mathrm{CO}$ and $\mathrm{CO}_{2}$ in the catalyst were calculated by the numerical integration. A comparison with the experiment showed that all the absolute values of the relative error were less than $10 \%$. The simulation results showed that decreasing reaction temperature and catalyst diameter were conducive to reduce the influence of the internal diffusion on the methanol synthesis.
\end{abstract}

Keywords: global kinetics, internal effectiveness factor, diffusion-reaction model, methanol synthesis.

\section{INTRODUCTION}

Methanol has many great advantages in properties and its practical application as an alternative fuel ${ }^{1}$. Large scale methanol synthesis is the trend of the methanol industry in the world and corresponding methanol synthesis reactor technology is necessary for large scale methanol production ${ }^{2,3}$. When one designs methanol synthesis reactors, the mass transfer and reactions in a gas-solid catalyst are important in sizing the reactor. It is necessary to develop a method for studying of the reaction-diffusion process in catalyst for optimization of catalyst size and reactor design. The mathematical model of the internal effectiveness factor should be developed according to the property of multicomponent reaction-diffusion in the catalyst particle ${ }^{4}$.

Through the reaction-diffusion model, one can get the information about temperature and concentration gradients and judge if there is a dead zone in the catalyst particle. Scholars put great effort to the study of reaction-diffusion process and developed many different models based on different assumptions ${ }^{5-9}$. Although the two-dimensional mathematical model can describe both the axial and radial diffusion-reaction process of cylinder catalyst ${ }^{10-12}$, it can hardly be extended equally to other irregular catalyst. However, one-dimensional model with the advantages of reliable and easy to solve, has been widely employed in reactor design. Zhu et al. ${ }^{27}$ proposed a multicomponent reaction-diffusion mathematical model for internal effectiveness factor of a regular shift reaction catalyst, which was sphericized according to the equivalent specific outer surface area ${ }^{13-14}$. Li et al.$^{34}$ also established a one-dimensional isothermal multicomponent reaction-diffusion model for irregularly shaped ammonia synthesis catalyst $\mathrm{A} 301^{15}$. It is suggested that spherical form of the catalyst could be extended equally to other irregular catalyst to optimize catalyst structure and reactor design. Permikin ${ }^{16}$ studied the concentration distribution and surface reacted layer growth. Lommerts ${ }^{17}$ did a great deal of work to describe the effects of internal mass transport limitations in methanol synthesis.

Methanol synthesis catalyst SC309 is a kind of $\mathrm{Cu}-$ -based catalyst and successfully used in methanol syn- thesis plants in China. It has higher activity under lower temperature until $180^{\circ} \mathrm{C}$ and is qualified for application in large-scale plants. But few researchers gave insight into the performance of this catalyst and none of the previous work from the literature was concerned with verification of the model of the internal effectiveness factor of SC309 by experiments.

The present work aims to develop a one-dimension isothermal multicomponent reaction-diffusion model for methanol synthesis with commercial catalyst SC309 to describe the coupled reaction and diffusion process in the catalyst. The numeric solution of the internal effectiveness factor of the catalyst was gained based on the model above. The model was verified by the global reaction rate obtained in a gradientless reactor_with satisfactory results. The effects of internal diffusion on the methanol synthesis reaction over commercial catalyst were investigated. The integrative methodology developed in this study can also be extended equally to other irregular catalyst to optimize catalyst structure and reactor design.

\section{EXPERIMENTAL}

\section{Intrinsic kinetic model}

Three reactions possibly occurred in methanol synthesis: hydrogenation of both carbon monoxide and carbon dioxide and water-gas shift reaction, which were as follows: $\mathrm{CO}+2 \mathrm{CO}_{2} \Leftrightarrow \mathrm{CH}_{3} \mathrm{OH}$

$\mathrm{CO}+3 \mathrm{H}_{3} \Leftrightarrow \mathrm{CH}_{3} \mathrm{OH}+\mathrm{H}_{2} \mathrm{O}$

$\mathrm{CO}+\mathrm{H}_{2} \mathrm{O} \Leftrightarrow \mathrm{CO}_{2}+\mathrm{H}_{2}$

The intrinsic kinetic expressions used in present work were gained from our previous study ${ }^{18}$, as listed below. Graaf et al. ${ }^{19-20}$ also did a great deal of work on methanol synthesis, and achieved significant results about kinetics and internal diffusion.

$$
\begin{aligned}
& r_{C O}=-\frac{d N_{C O}}{d W}=\frac{k_{1} f_{C O} f_{\mathrm{H}_{2}}^{2}\left(1-\beta_{1}\right)}{\left(1+K_{\mathrm{CO}} f_{C O}+K_{\mathrm{CO}_{2}} f_{\mathrm{CO}_{2}}+K_{\mathrm{H}_{2}} f_{\mathrm{H}_{2}}{ }^{3}\right)} \\
& r_{\mathrm{CO}_{2}}=-\frac{d N_{\mathrm{CO}_{2}}}{d W}=\frac{k_{2} f_{\mathrm{CO}_{2}} f_{\mathrm{H}_{2}}^{3}\left(1-\beta_{2}\right)}{\left(1+K_{\mathrm{CO}} f_{\mathrm{CO}}+K_{\mathrm{CO}_{2}} f_{\mathrm{CO}_{2}}+K_{\mathrm{H}_{2}} f_{\mathrm{H}_{2}}{ }^{4}\right)}
\end{aligned}
$$


$k_{1}=0.5616 \times 10^{3} \exp \left(-2.1546 \times 10^{4} / R_{g} T\right)$

$k_{2}=3.139 \times 10^{3} \exp \left(-3.3766 \times 10^{4} / R_{g} T\right)$

$K_{C O}=\exp \left[1.3945+1.9103 \times 10^{3} \times(1 / T-1 / \bar{T})\right]$

$K_{\mathrm{CO}_{2}}=\exp \left[0.0625+1.7463 \times 10^{4} \times(1 / T-1 / \bar{T})\right]$

$K_{H_{3}}=\exp \left[0.3984+3.9912 \times 10^{3} \times(1 / T-1 / \bar{T})\right]$

where $\beta_{1}=\frac{f_{\mathrm{CH}_{3} \mathrm{OH}}}{K_{f_{1}} f_{\mathrm{CO}} f_{\mathrm{H}_{2}}^{2}}, \beta_{2}=\frac{f_{\mathrm{CH}_{3} \mathrm{OH}} f_{\mathrm{H}_{2} \mathrm{O}}}{K_{f_{2}} f_{\mathrm{CO}_{2}} f_{\mathrm{H}_{2}}^{3}}, \bar{T}=490.71 K$.

\section{Global reaction rate}

Global reaction rate for methanol synthesis was measured in the apparatus, as shown in Figure 1. The feed gas consisted of $\mathrm{CO}, \mathrm{CO}_{2}, \mathrm{H}_{2}$ and inert gases $\mathrm{N}_{2}$. Traces of $\mathrm{O}_{2}$ were removed by oxygen remover at about $423 \mathrm{~K}$.

A magnetically driven internal-recycle gradientless reactor was used, whose impeller speed was in the range of 2500-3000 r/min. The impeller speed was high enough to eliminate the influence of external diffusion when $>2500$ $\mathrm{rpm}$. The catalyst bed was set in the isothermal zone.

SC309 is a new type methanol synthesis catalyst of Beijing Sanju Environmental Protection and New Material Co., Ltd. The Weight Ratio (\%) is: $\mathrm{Cu} / \mathrm{Zn} / \mathrm{Al}=69: 23: 8$ analyzed by Inductively Coupled Plasma Atomic Emissios Spetrometuy (ICP-AES). Six cylindrical catalyst pellets were packed in the reactor and the particle size was $\Phi$ $5 \mathrm{~mm} \times 5 \mathrm{~mm}$. During operation, the temperature difference between inlet and outlet gas was less than $0.5^{\circ} \mathrm{C}$.

The operating conditions were as follows: temperature ranging from $180-260^{\circ} \mathrm{C}$, pressure $4-8 \mathrm{MPa}$, and space velocity $4000-10000 \mathrm{~h}^{-1}$. Global reaction rate was uniquely determined by effective diffusivity under specific external conditions, and the results are listed in Table 1.

\section{Characterization of catalyst}

XRD pattern of fresh calcined $\mathrm{Cu}-\mathrm{Al}_{2} \mathrm{O}_{3}$ catalyst is shown in Figure 2 . The diffraction peaks at $35.5^{\circ}(11-1)$, $38.7^{\circ}\left(\begin{array}{lll}1 & 1 & 1\end{array}\right)$ and $48.7^{\circ}(20-2)$ were attributed to distinct peaks of monoclinic $\mathrm{CuO}$ (JCPDS 48-1548). Moreover,

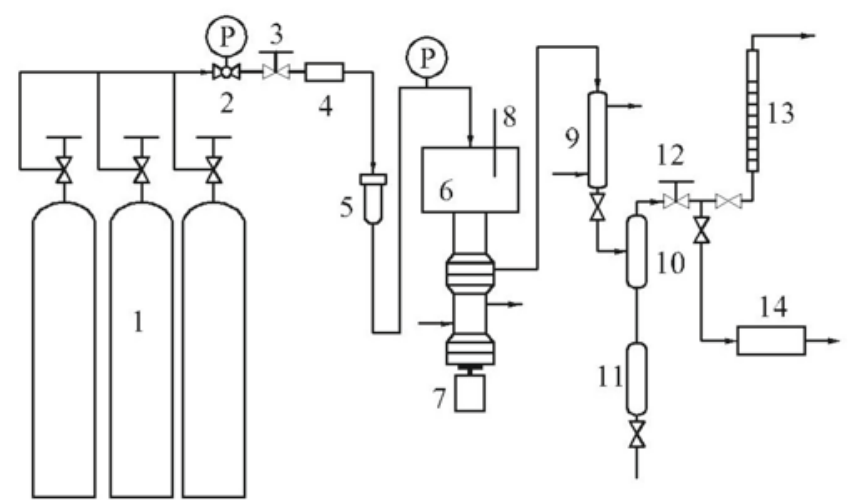

1 - gas cylinder; 2 - pressure regulating valve; 3 - needle valve; 4 - mass flow controller; 5 - oxygen remover; 6 - internal-recycle gradientless reactor; 7 - magnetic stirrers; 8 - thermocouple; 9 - condenser; 10 - gas-liquid separator; 11 - liquid storage tank; 12 - back pressure valve; 13 - soap bubble flow meter; 14 - gas chromatograph

Figure 1. Flowchart for experimental determination of global reaction rates

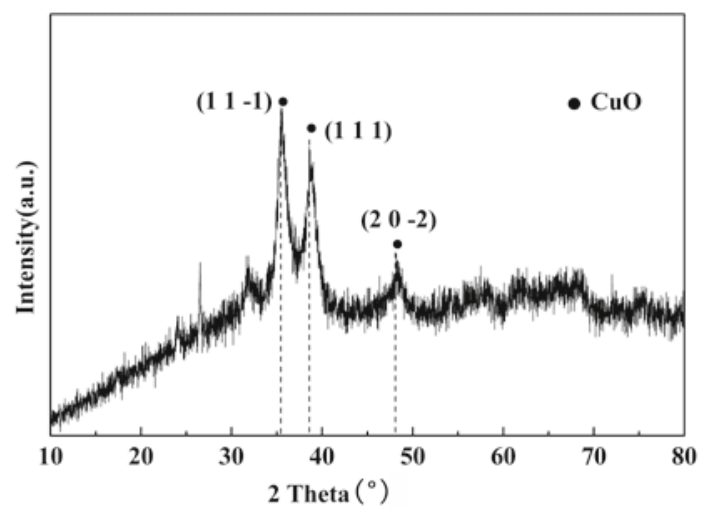

Figure 2. XRD pattern of catalyst SC309

the average crystal diameter of $\mathrm{CuO}$ is $40.4 \mathrm{~nm}$ which was estimated from $(20-2)$ plane using Scherrer equation ${ }^{21}$.

The $\mathrm{N}_{2}$ adsorption/desorption isotherms and $\mathrm{BJH}$ pore size distribution curve of SC309 are presented in Figure 3. This one indicated that the catalyst exhibited a type IV isotherm attributed to mesoporous structure. The surface area $\left(\mathrm{S}_{\mathrm{BET}}\right)$ is $103.0 \mathrm{~m}^{2} \cdot \mathrm{g}^{-1}, \mathrm{BJH}$ desorption pore volume $\left(\mathrm{V}_{\mathrm{P}}\right)$ is $0.293 \mathrm{~cm}^{3} \cdot \mathrm{g}^{-1}$ and $\mathrm{BJH}$ desorption average pore diameter $\left(D_{P}\right)$ is $8.8 \mathrm{~nm}$. Figure $3(\mathrm{~b})$ showed

Table 1. Experiment data of the global kinetics of methanol synthesis

\begin{tabular}{|c|c|c|c|c|c|c|c|c|c|c|c|}
\hline \multirow{2}{*}{ No. } & \multirow{2}{*}{$\frac{P}{/ \mathrm{MPa}}$} & \multirow{2}{*}{$\frac{t}{I^{\circ} \mathrm{C}}$} & \multirow{2}{*}{$\frac{N_{\text {in }}}{/ \mathrm{mol} \cdot \mathrm{h}^{-1}}$} & \multicolumn{3}{|c|}{$y_{i, i n}$} & \multicolumn{3}{|c|}{$y_{i, \text { out }}$} & \multicolumn{2}{|c|}{$r_{\text {global }} / \mathrm{mol} \cdot \mathrm{g}^{-1} \cdot \mathrm{h}^{-1}$} \\
\hline & & & & $\mathrm{H}_{2}$ & $\mathrm{CO}$ & $\mathrm{CO}_{2}$ & $\mathrm{H}_{2}$ & $\mathrm{CO}$ & $\mathrm{CO}_{2}$ & $\mathrm{CO}$ & $\mathrm{CO}_{2}$ \\
\hline 1 & 8.00 & 200.0 & 0.2104 & 0.6139 & 0.1146 & 0.0949 & 0.5623 & 0.0832 & 0.1181 & 1.22E-02 & 1.55E-03 \\
\hline 2 & 6.04 & 239.6 & 0.1649 & 0.6139 & 0.1146 & 0.0949 & 0.5207 & 0.0887 & 0.1296 & 1.10E-02 & $1.45 \mathrm{E}-03$ \\
\hline 4 & 5.00 & 200.0 & 0.1925 & 0.6139 & 0.1146 & 0.0949 & 0.5626 & 0.0878 & 0.1178 & 9.94E-03 & 1.07E-03 \\
\hline 5 & 5.02 & 240.0 & 0.3253 & 0.6139 & 0.1146 & 0.0949 & 0.5501 & 0.1007 & 0.1185 & 1.31E-02 & 1.47E-03 \\
\hline 6 & 4.02 & 259.8 & 0.3562 & 0.6139 & 0.1146 & 0.0949 & 0.5496 & 0.1237 & 0.1135 & $4.28 \mathrm{E}-03$ & $3.14 \mathrm{E}-04$ \\
\hline 7 & 8.03 & 180.1 & 0.3292 & 0.5821 & 0.0857 & 0.0290 & 0.5385 & 0.0813 & 0.0328 & 4.57E-03 & $1.16 \mathrm{E}-04$ \\
\hline 8 & 7.00 & 180.0 & 0.2154 & 0.5821 & 0.0857 & 0.0290 & 0.5407 & 0.0751 & 0.0329 & $4.28 \mathrm{E}-03$ & 1.25E-04 \\
\hline 9 & 7.02 & 220.1 & 0.3494 & 0.5821 & 0.0857 & 0.0290 & 0.5338 & 0.0512 & 0.0323 & 1.64E-02 & 1.24E-03 \\
\hline 10 & 7.01 & 260.1 & 0.2439 & 0.5821 & 0.0857 & 0.0290 & 0.5130 & 0.0621 & 0.0335 & 9.76E-03 & 7.92E-04 \\
\hline 11 & 6.02 & 200.1 & 0.3179 & 0.5821 & 0.0857 & 0.0290 & 0.5468 & 0.0622 & 0.0324 & 1.03E-02 & 4.97E-04 \\
\hline 12 & 4.01 & 260.3 & 0.2944 & 0.5821 & 0.0857 & 0.0290 & 0.5248 & 0.0758 & 0.0326 & 4.82E-03 & 4.32E-04 \\
\hline 13 & 6.02 & 200.4 & 0.1472 & 0.7199 & 0.0655 & 0.0258 & 0.6808 & 0.0364 & 0.0303 & $6.23 \mathrm{E}-03$ & 5.15E-04 \\
\hline 14 & 6.02 & 180.2 & 0.4023 & 0.7199 & 0.0655 & 0.0258 & 0.7016 & 0.0624 & 0.0281 & $3.25 \mathrm{E}-03$ & 1.02E-04 \\
\hline 15 & 5.00 & 180.0 & 0.2870 & 0.7199 & 0.0655 & 0.0258 & 0.6982 & 0.0612 & 0.0286 & $2.94 \mathrm{E}-03$ & $9.14 \mathrm{E}-05$ \\
\hline 17 & 4.02 & 240.2 & 0.2598 & 0.7199 & 0.0655 & 0.0258 & 0.7026 & 0.0407 & 0.0267 & 8.42E-03 & 1.02E-03 \\
\hline 18 & 4.00 & 220.1 & 0.3151 & 0.7199 & 0.0655 & 0.0258 & 0.7066 & 0.0391 & 0.0272 & 1.03E-02 & 1.00E-03 \\
\hline 19 & 4.01 & 200.1 & 0.3859 & 0.7199 & 0.0655 & 0.0258 & 0.7087 & 0.0521 & 0.0277 & $6.80 \mathrm{E}-03$ & 3.89E-04 \\
\hline
\end{tabular}



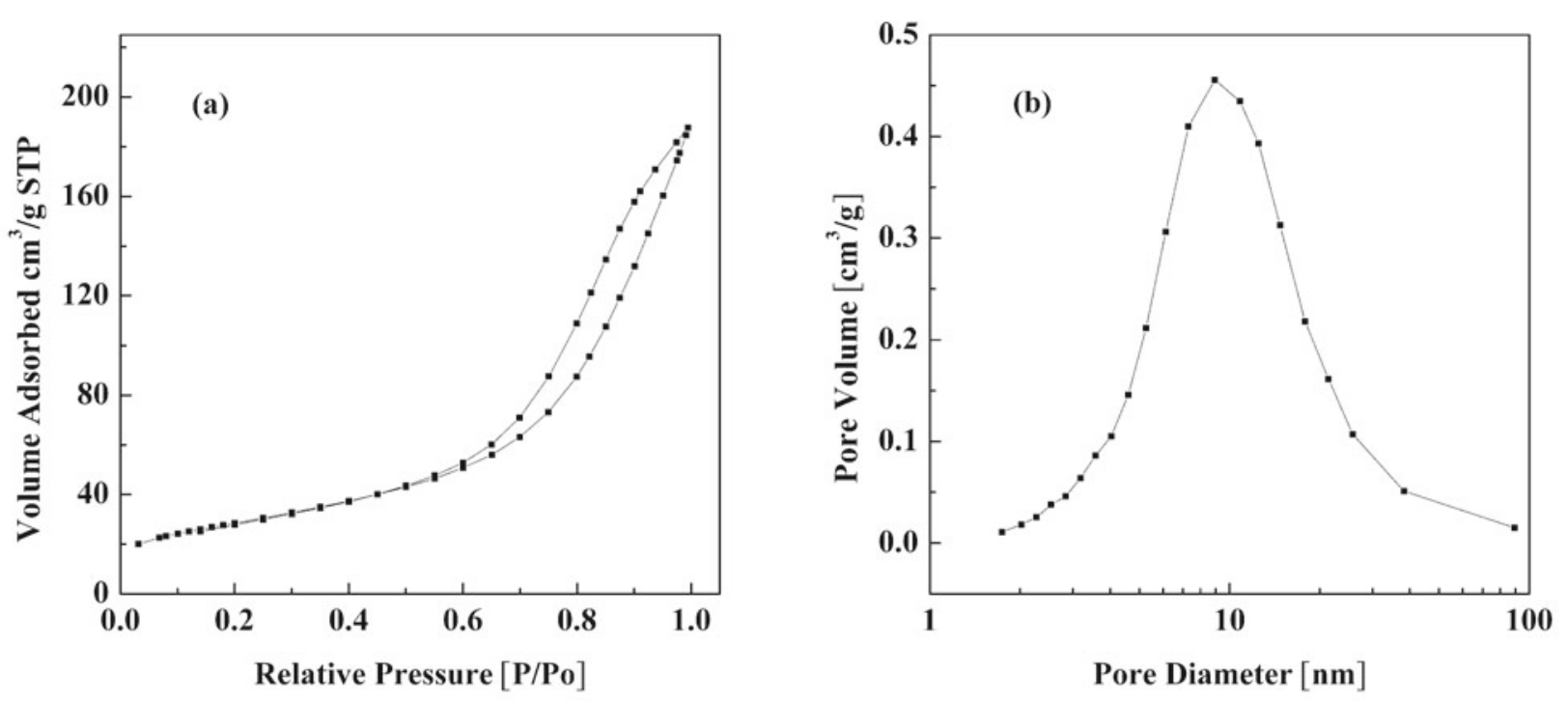

Figure 3. (a) $\mathrm{N}_{2}$ adsorption/desorption isotherms and (b) BJH pore-size distribution

the pore size distribution of the catalyst concentrated around $10 \mathrm{~nm}$.

\section{DIFFUSION-REACTION MODEL DESCRIPTION}

The present investigation focused on the interaction between diffusion and reaction inside a cylindrical catalyst pellet, which had so far received only limited attention $^{22-23}$.

Since the pores in the pellets were not straight and cylindrical, rather, they were a series of tortuous and interconnecting paths of pore bodies and pore throats with varying cross-sectional $\operatorname{areas}^{\mathbf{2 4}}$. In this study, the parallel cross-linked pore model ${ }^{25}$ was used to describe the mass transfer process of the multi-component diffusion system in the catalyst.

Diffusion and reaction in composite catalyst particles were calculated using the dust-gas-model ${ }^{26}$, and the diffusion flux of components $i$ in the catalyst pores can be described as:

$G_{i}=G_{i}^{D}+G_{i}^{V}$

$G_{i}^{D}$ is consist of molecular diffusion and Knudsen diffusion. Stagnation flux $G_{i}^{V}$ can be calculated as: $G_{i}^{V}=-\frac{y_{i} B_{0} P}{R_{g} T \mu} \frac{d P}{d x}$, where $B_{0}=r_{a}^{2} / 8$

Since the pore radius of the commercial catalyst was far less than $1 \mu \mathrm{m}$, stagnation flux could be neglected. The diffusion flux of reactants could be expressed as Equation (7), then derivative of Equation (7) and we had Equation (8).

$G_{i}=D_{e f f, i}\left(\frac{P}{Z R_{g} T}\right) \frac{d y_{i}}{d R}$

$\frac{d G_{i}}{d R}=\left(\frac{P}{Z R_{g} T}\right)\left[D_{e f f, i} \frac{d^{2} y_{i}}{d R^{2}}+\left(\frac{d D_{e f f, i}}{d R}\right)\left(\frac{d y_{i}}{d R}\right)\right]$

For a spherical porous catalyst in multicomponent reaction system, a steady-state material balance on a reaction component $i$ as it entered, left, and reacted in a spherical shell of inner radius $R$ and outer radius $R+d R$ of the pellet was performed. Combined with the intrinsic kinetics equation, the one-dimensional isothermal reaction-diffusion model, actually, a material balance equation used to describe the process of reaction and diffusion of all reaction species in catalyst could be developed and the model equation was shown in Equation (9).

$\frac{d^{2} y_{i}}{d R^{2}}+\left[\frac{2}{R}+\left(\frac{d D_{e f f, i}}{d R}\right) / D_{e f f, i}\right] \frac{d y_{i}}{d R}=\left(\frac{Z R_{g} T}{P}\right)\left(\frac{1}{D_{e f f, i}}\right) \rho_{P} r_{i}$

Substituting specific radius $x=R / R \mathrm{p}$ into Equation (9), and we had Equation (10).

$\frac{d^{2} y_{i}}{d R^{2}}+\left[\frac{2}{R}+\left(\frac{d D_{e f f, i}}{d R}\right) / D_{\text {eff }, i}\right] \frac{d y_{i}}{d R}=\left(\frac{Z R_{g} T}{P}\right)\left(\frac{1}{D_{e f f, i}}\right) \rho_{P} r_{i}$

The effective diffusivity of components $i$ of gas mixture in the catalyst pores was ${ }^{15,27}$ :

$\frac{d^{2} y_{i}}{d x^{2}}+\left[\frac{2}{x}+\left(\frac{d D_{e f f, i}}{d x}\right) / D_{e f f, i}\right] \frac{d y_{i}}{d x}=\left(\frac{Z R_{g} T}{P}\right)\left(\frac{R_{P}^{2}}{D_{e f f, i}}\right) \rho_{P} r_{i}$

The Knudsen diffusion coefficients ${ }^{28}$ of components $i$ were calculated from Stefan-Maxwell ${ }^{29}$ equation: $D_{k i}=9.7 \times 10^{3} r_{a} \sqrt{T / M_{i}}$

Therefore, the binary diffusion coefficients between each component in gas were estimated by the Fuller, Schettler and Giddings' (FSG) equation ${ }^{30}$ :

$D_{i j}=\frac{0.001 \times 0.101325 T^{1.75}\left(1 / M_{i}+1 / M_{j}\right)^{0.5}}{P\left[\left(\sum V\right)_{i}^{1 / 3}+\left(\sum V\right)_{j}^{1 / 3}\right]^{2}}$

$\left(\sum V_{i}\right)$ was the molecule diffusion volume, the values could be found in reference ${ }^{30}$.

The model established based on the classical simplifying assumptions included:

- The flow in the monolith channel that was laminar, fully developed and had one direction only, and the gas phase that was considered to be incompressible and steady.

- Pressure drop along the monolith channel that was small and negligible ${ }^{31}$; convective flux in the monolith wall that was irrelevant and hence not considered ${ }^{32}$. 
- The cylinder catalyst SC309 with the size of $\Phi 5 \mathrm{~mm}$ $\times 5 \mathrm{~mm}$ was equivalent to a spherical catalyst whose diameter was $5 \mathrm{~mm}$, and the catalyst was isothermal.

- According to the parallel cross-linked pore model, the molecular diffusion coefficient should be calculated based on the composition of the catalyst outer surface, the average pore radius should be used in the calculation of Knudsen diffusion coefficient.

To ensure computational accuracy, $\mathrm{CO}$ and $\mathrm{CO}_{2}$ were chosen as the key components to calculate effective diffusivity because of the lowest values of their mole fraction.

So, the model equations were:

$\frac{d^{2} y_{C O}}{d x^{2}}+\frac{2}{x} \frac{d y_{C O}}{d x}=\left(\frac{Z R_{g} T}{P}\right)\left(\frac{R_{P}^{2}}{D_{e f f, C O}}\right) \rho_{P} r_{C O}$

$\frac{d^{2} y_{\mathrm{CO}_{2}}}{d x^{2}}+\frac{2}{x} \frac{d y_{\mathrm{CO}_{2}}}{d x}=\left(\frac{Z R_{g} T}{P}\right)\left(\frac{R_{P}^{2}}{D_{\text {eff }, \mathrm{CO}}}\right) \rho_{P} r_{C O}$

With boundary conditions:

$x=1, y_{\mathrm{CO}}=y_{\mathrm{CO}, \mathrm{s}}, y_{\mathrm{CO}_{2}}=y_{\mathrm{CO}_{2}, \mathrm{~s}}$

$x=0, \frac{d y_{C O}}{d x}=0, \frac{d y_{\mathrm{CO}_{2}}}{d x}=0$

The effectiveness factors of key component $\mathrm{CO}$ and $\mathrm{CO}_{2}$ could be indicated as the following equations ${ }^{24,27}$ :

$\zeta_{C O}=\frac{4 \pi R_{P}^{2} D_{e f f, C O}\left(d y_{C O} / d R\right)_{s}}{\frac{4}{3} \pi R_{P}^{3} \rho_{P} r_{C O, s}}\left(\frac{P}{Z R_{g} T}\right)$

$\zeta_{\mathrm{CO}_{2}}=\frac{4 \pi R_{P}^{2} D_{e f f, \mathrm{CO}_{2}}\left(d y_{\mathrm{CO}_{2}} / d R\right)_{s}}{\frac{4}{3} \pi R_{P}^{3} \rho_{P} r_{\mathrm{CO}_{2}, s}}\left(\frac{P}{Z R_{g} T}\right)$

The diffusion-reaction model is a set of second order ordinary differential equations with boundary-value problems (ODE-BVPs). The intrinsic kinetics model used in this work is a hyperbolic kinetics model, which is a function of fugacity $f$. Meanwhile, the fugacity $f$ is a function of concentration $y$ which depends from $\mathrm{R}$ or $\mathrm{x}$. Thus the diffusion-reaction model does not have analytical solution. Numerical methods such as nume- rical integration shooting method, weighted allowance method, finite element method, finite difference method, singular perturbation method, and orthogonal collocation method are often used to solve ODEs. The numerical integration shooting method ${ }^{33-34}$ is a high precision and strong practical method, and it solves ODE-BVPs by converting boundary-value problem (BVP) to initial-value problem (IVP).

The model equations were solved based on experi$\frac{d y_{\mathrm{CO}}}{d x} \quad \frac{d y_{\mathrm{CO}_{2}}}{d x}$ mental data of global reaction rate. $\frac{d x}{d x}$ and $\frac{d y_{\mathrm{O}_{2}}}{d x}$ were obtained by using numerical integration shooting method via solving Equations (13) and (14), then substituting $\frac{d y_{\mathrm{CO}}}{d x}$ and $\frac{d y_{\mathrm{CO}_{2}}}{d x}$ into Equations (15) and (16). Then the effectiveness factors of the catalyst $\zeta_{C O}$ and $\zeta_{\mathrm{CO}_{2}}$ were obtained.

\section{RESULTS AND DISCUSSION}

\section{Validation of the reaction-diffusion model}

The reaction-diffusion model Equations (13)-(14) were used together with the intrinsic kinetics Equations (4)-(5) to simulate the behavior.

The calculated internal effectiveness factors $\zeta_{C O, c a l}$ and $\zeta_{\mathrm{CO}_{2}, \text { cal }}$ of the catalyst under different experimental global kinetics conditions were computed based on solving model equations. The experimental internal effectiveness factors $\zeta_{\mathrm{CO} \text {,exp }}$ and $\zeta_{\mathrm{CO}_{2} \text {,exp }}$ can be obtained as follows:

$\zeta_{C O, \text { exp }}=\frac{r_{C O, \text { global }}}{r_{C O, \text {,intrinsic }}}$

$\zeta_{\mathrm{CO}_{2}, \text { exp }}=\frac{r_{\mathrm{CO}_{2}, \text { global }}}{r_{\mathrm{CO}_{2}, \text { intrinsic }}}$

Experimental global rates $r_{\text {global }}$ were calculated according to mass balance at the inlet and the outlet of the reactor:

$r_{C O, \text { global }}=-\frac{N_{\text {out }} \times y_{C O, \text { out }}-N_{\text {in }} \times y_{C O, \text { in }}}{W}$

Table 2. Calculation results and experimental value of $\zeta_{\mathrm{CO}}$ and $\zeta_{\mathrm{CO}_{2}}$

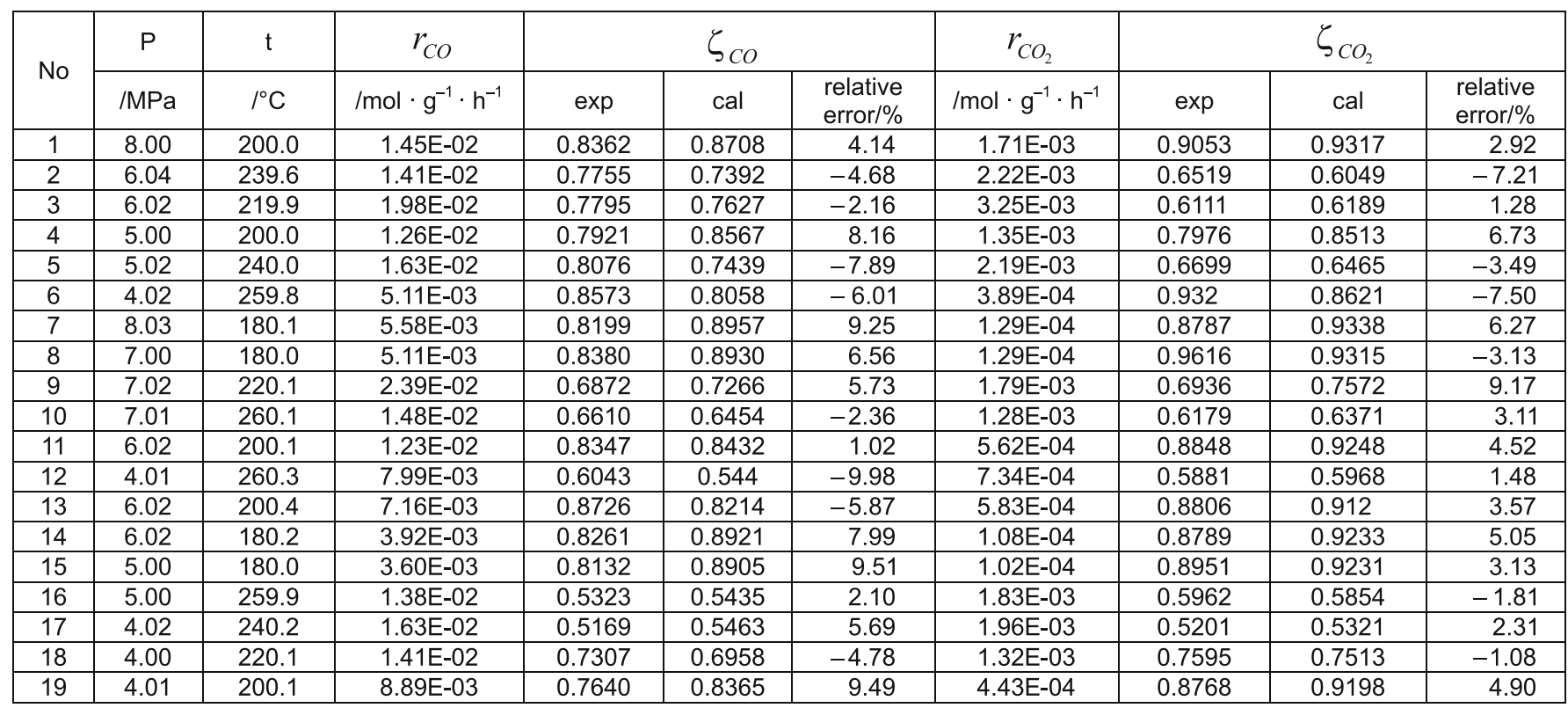


$r_{\mathrm{CO}_{2}, \text { global }}=-\frac{N_{\text {out }} \times y_{\mathrm{CO}_{2}, \text { out }}-N_{\text {in }} \times y_{\mathrm{CO}_{2}, \text { in }}}{W}$

relative error $=\frac{\zeta_{c a l}-\zeta_{\exp }}{\zeta_{\exp }} \times 100 \%$

Where $y_{i, \text { in }}$ and $y_{i, \text { out }}$ were mole fractions of component $i$ at the inlet and the outlet of the reactor, respectively.

The results of reaction rates and the comparison of the calculated values with experimental values of internal effectiveness factor are presented in Table 2.

On analysis of the results obtained, the calculated values from the model were in agreement with the experimental values on the whole. As the actual diffusion channels of the cylindrical catalyst were shorter than those of the sphericized particles, most of the experimental values were smaller than the model values. Even then it could also be concluded that the model developed on the multicomponent reaction-diffusion in a cylindrical catalyst was reliable and could be used to describe the concentration distributions in the catalyst.

The simulations gave the internal concentration profiles of $\mathrm{CO}$ and $\mathrm{CO}_{2}$ which are displayed in Figure 4.

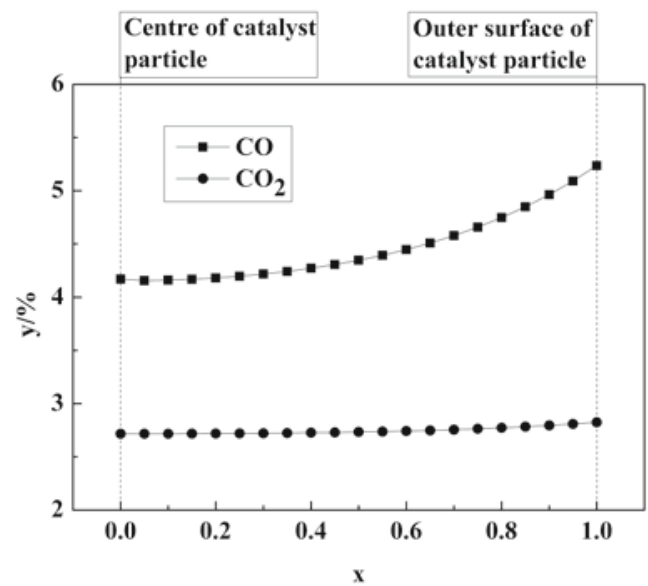

Figure 4. Concentration profile in catalyst pores at condition No.10

Figure 4 indicates that the $\mathrm{CO}$ concentration decreased quickly near the surface of the cylindrical catalyst, but decreased slowly near the center of the catalyst. The $\mathrm{CO}_{2}$ concentration almost remained the same. It can be ascribed to that the reaction rate of $\mathrm{CO}$ hydrogenation near the surface was higher than that near the centre of the cylindrical catalyst. Table 1 shows that the $\mathrm{CO}_{2}$ reaction rate was much lower than that of $\mathrm{CO}$, which may be due to $\mathrm{CO}_{2}$ concentrating in the cylindrical catalyst. As a whole, the reaction of methanol synthesis near the cylindrical catalyst surface was faster than that near the centre of the cylindrical catalyst. Finally, the exact values of the effectiveness factors of the components could be obtained from these calculations.

\section{Effect of temperature}

The simulated conditions were as follows: the concentrations at the surface of the catalyst were $\mathrm{H}_{2} 0.5207$, $\mathrm{CO} 0.0761, \mathrm{CO}_{2} 0.0348, \mathrm{CH}_{3} \mathrm{OH} 0.0453, \mathrm{H}_{2} \mathrm{O} 0.0055$ and $\mathrm{N}_{2} 0.3225$, pressure $6 \mathrm{MPa}$, the diameter of the catalyst $5 \mathrm{~mm}$. The results are shown in Figure 5.

Figure 5 indicates that the diffusion effectiveness factors of $\mathrm{CO}$ and $\mathrm{CO}_{2}$ decreased with the increase of reaction

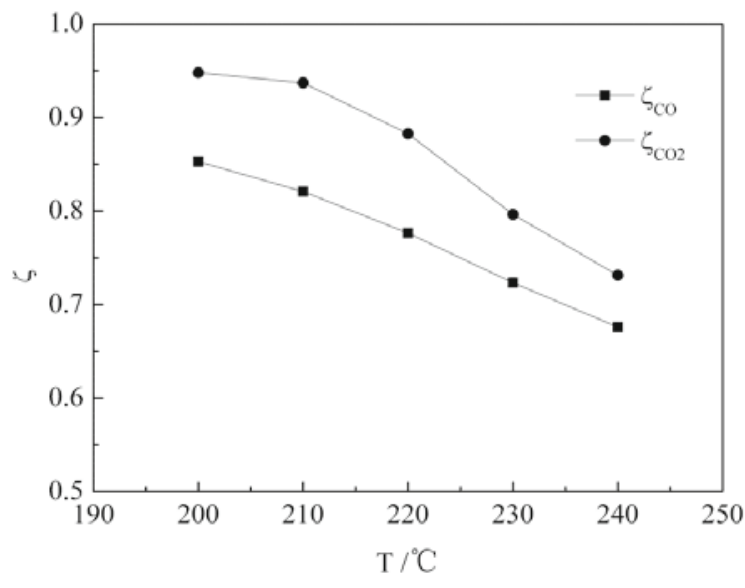

Figure 5. Effect of the temperature on $\zeta_{\mathrm{CO}}$ and $\zeta_{\mathrm{CO}_{2}}$

temperature, while both reaction rate and diffusion coefficient increased with the rising temperature in the experimental range. However, it was demonstrated that the effect on the intrinsic reaction rate by temperature was more significant compared to the internal diffusion coefficient counterpart. According to Equations (15) and (16), the diffusion effectiveness factors inevitably decreased with the rising temperature. The result indicated that there existed an optimum reaction temperature between reaction rate and diffusion coefficient.

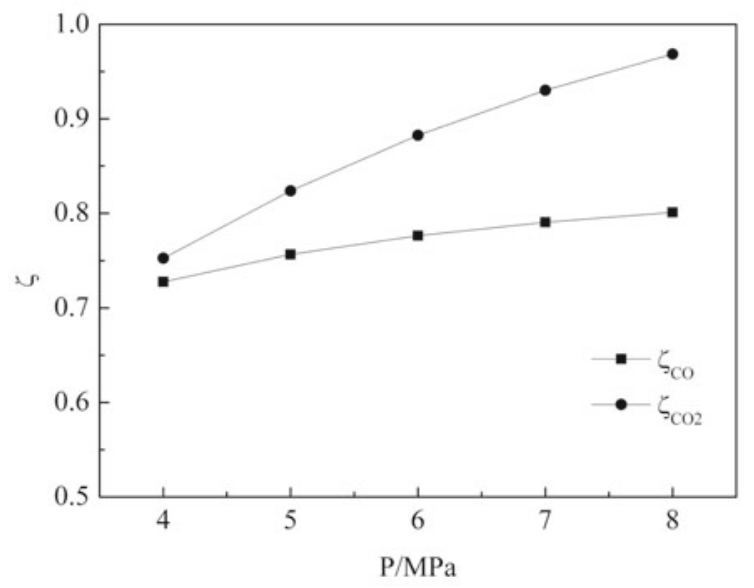

Figure 6. Effect of the pressure on $\zeta_{\mathrm{CO}}$ and $\zeta_{\mathrm{CO}_{2}}$

\section{Effect of pressure}

The simulated conditions were as follows: the concentrations at the surface of the catalyst were $\mathrm{H}_{2} 0.5207, \mathrm{CO}$ 0.0761, $\mathrm{CO}_{2} 0.0348, \mathrm{CH}_{3} \mathrm{OH} 0.0453, \mathrm{H}_{2} \mathrm{O} 0.0055$ and $\mathrm{N}_{2}$ 0.3225 , temperature $220^{\circ} \mathrm{C}$, the diameter of the catalyst $5 \mathrm{~mm}$. The calculation results are shown in Figure 6.

Figure 6 presents $\zeta_{\mathrm{CO}}$ and $\zeta_{\mathrm{CO}_{2}}$ plotted as a function of pressure. On increasing the pressure from $4 \mathrm{MPa}$ to $8 \mathrm{MPa}$, a significant increase in diffusion effectiveness factor of $\mathrm{CO}_{2}$ was observed. This was due to a significant increase in the motive force resulting from pressure difference between catalyst inside and outside. Table 2 showed that $r_{c o}$ was greater than $r_{\mathrm{CO}_{2}}$ and the effect of the pressure on $r_{c o}$ was very significant, $r_{c o}$ increased monotonically with pressure ${ }^{18}$. As the pressure was increased, the influence of pressure on $\zeta_{C O}$ was less than the influence on $\zeta_{\mathrm{CO}_{2}}$. Therefore, increasing pressure was conducive to the methanol synthesis from syngas. 


\section{Effect of catalyst diameter}

The simulated conditions were as follows: the concen-

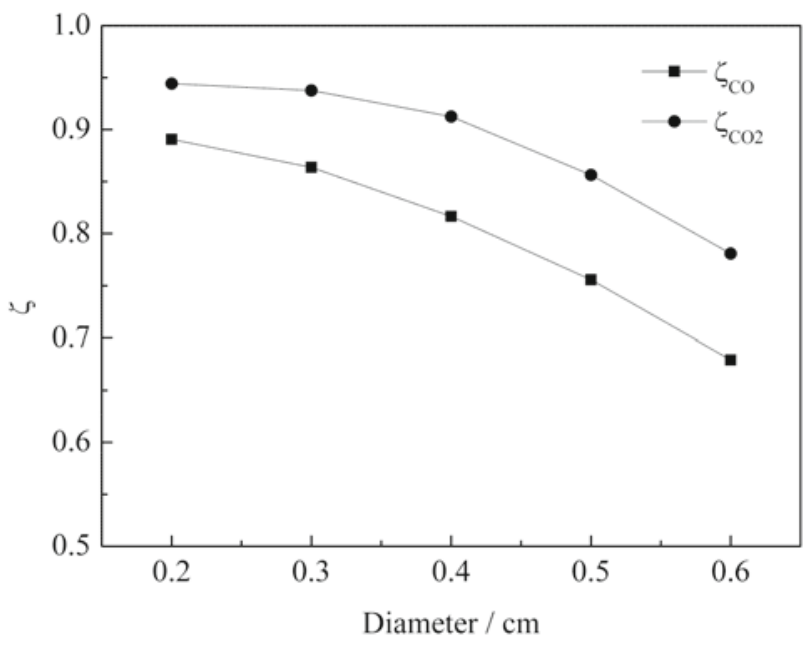

Figure 7. Effect of the catalyst diameter on $\zeta_{\mathrm{CO}}$ and $\zeta_{\mathrm{CO}_{2}}$

trations at the surface of the catalyst were $\mathrm{H}_{2} \quad 0.5207$, $\mathrm{CO} 0.0761, \mathrm{CO}_{2} 0.0348, \mathrm{CH}_{3} \mathrm{OH} 0.0453, \mathrm{H}_{2} \mathrm{O} 0.0055$ and $\mathrm{N}_{2} 0.3225$, pressure $7 \mathrm{MPa}$, temperature $230^{\circ} \mathrm{C}$. The calculation results are shown in Figure 7.

As given in Figure 7, the simulated values of $\zeta_{\mathrm{CO}}$ and $\zeta_{\mathrm{CO}_{2}}$ plotted against the catalyst diameter. Both $\zeta_{\mathrm{CO}}$ and $\zeta_{\mathrm{CO}_{2}}$ increased with the decrease of catalyst diameter. Based on the fact that diffusion flux increased by virtue of shorter monolith channel caused by decreasing size of catalyst particles, the factor of diffusion efficiency had weakened. As a result, the influence initiated by internal diffusion was very likely to be eliminated as long as the size of catalyst particle was small enough.

\section{CONCLUSIONS}

The global reaction rate was measured experimentally in the internal recycle gradientless reactor at $180-260^{\circ} \mathrm{C}$, 4-8 MPa and 4000-10 $000 \mathrm{~h}^{-1}$ over catalyst SC309.

The one-dimensional diffusion-reaction model for intra-particle diffusion limitations in methanol synthesis over catalyst SC309 was developed and the model was validated with independently obtained experimental methanol production data. All the absolute values of the relative error were less than $10 \%$ and the diffusion model in the present study proved to be accurate in describing the experimentally observed conversion rates for pressures up to $8 \mathrm{MPa}$ and temperatures up to $260^{\circ} \mathrm{C}$. Besides, it can also be used to analyze the diffusion-reaction process of the methanol synthesis catalyst.

The effect of different operation conditions on internal diffusion of methanol synthesis was analyzed and the results showed that decreasing reaction temperature and catalyst diameter were conducive to reduce the influence of the internal diffusion on the methanol synthesis.

\section{NOMENCLATURE}

$D_{\text {eff }} \quad-$ effective diffusion coefficient

$D_{k} \quad-$ Knudsen diffusion coefficient

$f^{k} \quad-$ fugacity, $\mathrm{MPa}$

$f(r) \quad$ - distribution function of pore radius

$G \quad-$ diffusion flux, $\mathrm{kmol} \cdot \mathrm{m}^{-2} \cdot \mathrm{h}^{-1}$
$K_{f} \quad-$ reaction equilibrium constant

$K \mathrm{i} \quad-$ adsorption equilibrium constant of species $i$

$k-\quad$ reaction rate constant

$M \quad$ - formula weight, $\mathrm{g} \cdot \mathrm{mol}^{-1}$

$N \quad-$ molar flow, $\mathrm{kmol} \cdot \mathrm{h}^{-1}$

$P \quad$ - pressure, $\mathrm{MPa}$

$R \quad$ - radius, $\mathrm{m}$

$R_{g} \quad$ - ideal gas constant, $8.314 \mathrm{~J} \cdot \mathrm{mol}^{-1} \cdot \mathrm{K}^{-1}$

$R_{p} \quad$ - equivalent diameter of catalyst, $\mathrm{m}$

$r \quad-$ reaction rate, $\mathrm{mol} \cdot \mathrm{g}^{-1} \cdot \mathrm{h}^{-1}$

$r_{a} \quad-$ pore radius, $\mathrm{cm}$

$T \quad$ - temperature, $\mathrm{K}$

$t \quad-$ temperature, ${ }^{\circ} \mathrm{C}$

$W \quad$ - weight of catalyst, $\mathrm{g}$

$x \quad-$ dimensionless radius

$y \quad-$ mole fraction

Z - compressibility factor

\section{Subscript}

cal - calculation results

exp - experimental results

global - global kinetics

in - reactor inlet

intrinsic - intrinsic kinetics

out - reactor outlet

$s \quad$ - outside surface of catalyst

\section{Greeks alphabet}

$\delta \quad-$ tortuosity

$\zeta \quad-$ internal diffusion effectiveness factor

$\theta \quad-$ porosity

$\mu \quad$ - viscosity, $\mathrm{Pa} \cdot \mathrm{s}$

$\varrho_{P} \quad-$ density, $\mathrm{kg} \cdot \mathrm{m}^{-3}$

\section{ACKNOWLEDGMENT}

The authors acknowledge financial support in this research from the National Key Technology R\&D Program of China (No. 2006BAE02B02).

\section{LITERATURE CITED}

1. Xie, K.C. \& Fang, D.Y. (2010). Methanol technology. Beijing, China: Chemical Industry Press. (Chinese reference)

2. Caulkin, R., Ahmad, A., Fairweather, M., Jia, X. \& Williams, R.A. (2007). An investigation of sphere packed shell-side columns using a digital packing algorithm. Comput. Chem. Eng. 31(12), 1715-1724. DOI: 10.1016/j.compchemeng.2007.03.014.

3. Ma, H.F., Ying, W.Y. \& Fang, D.Y. (2008). Simulation of a combined converter for methanol synthesis. J. East. China. U. Sci. Technol. 34, 149-153. from http://www.cnki.com.cn/ Article/CJFDTotal-HLDX200802000.htm (Chinese reference).

4. Aris, R. (1975). The mathematical theory of diffusion and reaction in permeable catalysts. London, UK: Clarendon Press.

5. Wood, J. \& Gladden, L.F. (2002). Modelling diffusion and reaction accompanied by capillary condensation using three-dimensional pore networks. Part 1. Fickian diffusion and pseudo-first-order reaction kinetics. Chem. Eng. Sci. 57(15), 3033-3045. DOI: 10.1016/S0009-2509(02)00183-5.

6. Wood, J., Gladden, L.F. \& Keil, F.J. (2002). Modelling diffusion and reaction accompanied by capillary condensation using three-dimensional pore networks. Part 2. Dusty gas model and general reaction kinetics. Chem. Eng. Sci. 57(15), 3047-3059. DOI: 10.1016/S0009-2509(02)00184-7.

7. Mariani, N.J., Mocciaro, C., Keegan, S.D., Martínez, O.M. \& Guillermo, F.B. (2009). Evaluating the effectiveness 
factor from a 1D approximation fitted at high Thiele modulus: Spanning commercial pellet shapes with linear kinetics. Chem. Eng. Sci. 64(11), 2762-2766. DOI: 10.1016/j.ces.2009.02.044.

8. Aumo, J., Wärnå, J., Salmi, T. \& Murzin, D.Y. (2006). Interaction of kinetics and internal diffusion in complex catalytic three-phase reactions: Activity and selectivity in citral hydrogenation. Chem. Eng. Sci. 61(2), 814-822. DOI: 10.1016/j. ces.2005.07.036.

9. Lee, J.K., Ko, J.B. \& Kim, D.H. (2004). Methanol steam reforming over $\mathrm{Cu} / \mathrm{ZnO} / \mathrm{Al}_{2} \mathrm{O}_{3}$ catalyst: kinetics and effectiveness factor. Appl. Catal., A. 278(1), 25-35. DOI: 10.1016/j. apcata.2004.09.022.

10. Guo, W.Y., Wu, W.Z., Luo, M. \& Xiao, W.D. (2013). Modeling of diffusion and reaction in monolithic catalysts for the methanol-to-propylene process. Fuel Process. Technol. 108, 133-138. DOI: 10.1016/j.fuproc.2012.06.005.

11. Pan, T.S. \& Zhu, B.C. (1998). Study on diffusion-reaction process inside a cylindrical catalyst pellet. Chem. Eng. Sci. 53(5), 933-946. DOI: 10.1016/S0009-2509(97)00385-0.

12. Zhang, L. Zhang, H.T., Ying, W.Y. \& Fang, D.Y. (2013). Dehydration of methanol to dimethyl ether over $\gamma-\mathrm{Al}_{2} \mathrm{O}_{3}$ catalyst: Intrinsic kinetics and effectiveness factor. Can. J. Chem. Eng. 91(9), 1538-1546. DOI: 10.1002/cjce.21760.

13. Zhu, B.C., Song, W.D., Fang, D.Y. \& Lu, D.Q. (1984). Multi-component diffusion model for effectiveness factor of porous catalyst (I) Multicomponent diffusion model and numerical computing method. J. Chem. Ind. Eng. 44, 33-40. from http://www.cnki.com.cn/Article/CJFDTotal-HGSZ198401003. htm (Chinese reference).

14. Zhu, B.C., Song, W.D., Fang, D.Y. \& Lu, D.Q. (1984). Multi-component diffusion model for effectiveness factor of porous catalyst (II) Effectiveness factor of high temperature slight reaction. J. Chem. Ind. Eng, 44, 41-50. form http://www. cnki.com.cn/Article/CJFDTotal-HGSZ198401004.htm (Chinese reference).

15. Li, T., Xu, M.S., Zhu, B.C., Fang, D.Y. \& Ying, W.Y. (2009). Reaction-diffusion model for irregularly shaped ammonia synthesis catalyst and its verification under high pressure. Ind. Eng. Chem. Res. 48(19), 8926-8933. DOI: 10.1021/ ie9001266.

16. Permikin, D.V. \& Zverev, V.S. (2013). Mathematical model on surface reaction diffusion in the presence of front chemical reaction. Int. J. Heat Mass Transfer. 57(1), 215-221. DOI: 10.1016/j.ijheatmasstransfer.2012.10.024.

17. Lommerts, B.J., Graaf, G.H. \& Beenackers, A.A.C.M. (2000). Mathematical modeling of internal mass transport limitations in methanol synthesis. Chem. Eng. Sci. 55(23), 5589-5598. DOI: 10.1016/S0009-2509(00)00194-9.

18. Lei, K., Ma, H.F., Zhang, H.T., Ying, W.Y. \& Fang, D.Y. (2013). Intrinsic kinetics of methanol synthesis over catalyst SC309. Nat. Gas. Chem. Ind. 3, 1-5. from http://www.cnki. com.cn/Article/CJFDTotal-TRQH201303000.htm (Chinese reference).

19. Graaf, G.H., Scholtens, H., Stamhuis, E.J. \& Beenackers A.A.C.M. (1990). Intra-particle diffusion limitations in low-pressure methanol synthesis. Chem. Eng. Sci. 45(4), 773-783. DOI: 10.1016/0009-2509(90)85001-T.

20. Graaf, G.H., Stamhuis, E.J. \& Beenackers, A.A.C.M. (1988). Kinetics of the low-pressure methanol synthesis. Chem. Eng. Sci. 43(12), 3185-3195. DOI: 10.1016/0009-2509(88)85127-3.

21. Patterson, A.L. (1939). The Scherrer Formula for X-Ray Particle Size Determination. Phys. Rev. 56(10), 978-982. DOI: 10.1103/PhysRev.56.978.

22. Aris, R. (1995). On shape factors for irregular particles-I: The steady state problem. Diffusion and reaction. Chem. Eng. Sci. 50(24), 3899-3903. DOI: 10.1016/0009-2509(96)81819-7.

23. Luss, D. \& Amundson, N.R. (1967). On a conjecture of Aris: proof and remarks. AlChE J. 13(4), 759-763. DOI: 10.1002/aic.690130431.
24. Fogler, H.S. (2005). Elements of Chemical Reaction Engineering. New Jersey, USA: Prentice-Hall International Inc.

25. Wheeler, A. (1950). Reaction rate and selectivity in catalyst pores (pp. 249-327). Adv. Catal.. Vol. New York, USA: Academic Press Inc.

26. Mason, E.A., Malinauskas, A.P. \& Evans, R.B. (1967). Flow and diffusion of gases in porous media. J. Chem. Phys. 46, 3199-3216. DOI: 10.1063/1.1841191.

27. Zhu, B.C. (2001). Chemical Reaction Engineering. Beijing, China: Chemical Industry Publishing Company. (Chinese reference)

28. Reid, R.C., Prausnitz, J.M. \& Poling, B.E. (1987). The properties of gases and liquids. New York, USA: McGraw Hill Book Co.

29. Curtiss, C.F. \& Hirschfelder, J.O. (1949). Transport properties of multicomponent gas mixture. J. Chem. Phys. 17, 553-555. DOI: 10.1063/1.1747319.

30. Fuller, E.N., Schettler, P.D. \& Giddings, J.C. (1966). A new method for prediction of binary gas-phase diffusion coefficients. Ind. Eng. Chem. 58(5), 18-27. DOI: 10.1021/ie50677a007.

31. Shah, R.K. \& London, A.L. (1978). Laminar Flow Forced Convection in Ducts. New York, USA: Academic Press.

32. Chen, J., Yang, H., Wang, N., Ring, Z. \& Dabros, T. (2008). Mathematical modeling of monolith catalysts and reactors for gas phase reactions. Appl. Catal. A. 345(1), 1-11. DOI: 10.1016/j.apcata.2008.04.010.

33. Kim, D.H. \& Lee, J. (2004). A robust iterative method of computing effectiveness factors in porous catalysts. Chem. Eng. Sci. 59(11), 2253-2263. DOI: 10.1016/j.ces.2004.01.056.

34. Lee, J. \& Kim, D.H. (2005). An improved shooting method for computation of effectiveness factors in porous catalysts. Chem. Eng. Sci. 60(20), 5569-5573. DOI: 10.1016/j. ces.2005.05.027. 